\title{
Innovation in orthopaedic surgery as it relates to evidence-based practice
}

\author{
M. Hofbauer • B. Muller $\cdot$ C. D. Murawski • \\ J. Karlsson · Freddie H. Fu
}

Published online: 4 January 2013

(c) Springer-Verlag Berlin Heidelberg 2013

\section{Introduction}

The field of medicine relentlessly pursues scientific advancement through ongoing basic science research and clinical investigation. In the present landscape of rapidly evolving technology in economic-driven medical systems, the interest and enthusiasm to innovate newer and more advanced products, diagnostic methods, less-expensive drugs and treatment methods has never been greater. Reitsma and Moreno, in their text titled Ethical Guidelines for Innovative Surgery, aptly describe this persistence "new is hot, old is not" [17, 18].

In the field of orthopaedics, millions benefit from medical innovations on a daily basis. The raise in multidisciplinary research between surgeons, basic scientists, bioengineers and product engineers, however, has resulted in numerous "innovations," of which not all have turned out to be beneficial to patients. We have learned repeatedly that new products, however, promising the preliminary studies, may ultimately prove to have no benefit. Further,

M. Hofbauer · B. Muller · C. D. Murawski · F. H. Fu ( $)$

Department of Orthopaedic Surgery, University of Pittsburgh

Medical Center, 3471 Fifth Avenue, Kaufman Building,

Suite 1011, Pittsburgh, PA 15213, USA

e-mail: ffu@upmc.edu

\section{Hofbauer}

Department of Trauma Surgery, Medical University Vienna,

Vienna, Austria

B. Muller · C. D. Murawski

Department of Orthopaedic Surgery, Academic Medical Center, Amsterdam, The Netherlands

J. Karlsson

Department of Orthopaedic Surgery, Sahlgrenska University

Hospital, Göteborg, Sweden hundreds of thousands of patients have experienced harm and complications as a result of new medical devices, drugs and therapies that lacked proper testing or inadequate scientific backing by clinical trials [24].

A common problem within the field of orthopaedics is that the motivation of the inventors (to improve health care) and manufacturers (to increase profit) to develop a new product may not completely align. Moreover, there is a lack of consensus on how "innovation" is defined. The April 2012 issue of Nature featured an article on rewardable innovation in drug therapy which reviewed nine articles that investigated the definition of innovation [2]. While the authors found that "newness" and "usefulness" were used in all but two studies, not a single study defined innovation as "has to be beneficial to patients" [2].

The purpose of this article is to emphasize that innovative medical devices and treatments must be safe, effective and economical but, above all, must result in no harm to patients. Moreover, new products should be judged critically by orthopaedic surgeons, scientists and industry prior to being utilized in standard clinical care. Furthermore, the following should be considered when evaluating innovative products: (1) the quality of the available scientific evidence, (2) potential conflicts of interest that may have influenced the presented evidence and (3) the influence that marketing may have on our evidence-based practice.

Quality of scientific evidence

Critically appraising available scientific evidence is often difficult and requires a certain level of training and/or experience. Newly developed products, however, can be presented to and become popular with the general public via marketing campaigns and media interest, often with 
inadequate basic scientific or clinical evidence to benefit patients. One such example in the 1980s and early 1990s was the use of synthetic artificial ligaments for ACL reconstruction that became highly attractive for orthopaedic surgeons as a means of avoiding the potential drawbacks associated with autografts and allografts [10]. Theoretically, artificial ligaments allow for a more aggressive rehabilitation compared to biologic grafts and provide a quick recovery and early return to sports [15]. Despite these encouraging short-term clinical benefits, subsequent clinical research revealed that the use of artificial ligaments was also associated with poor patient outcomes and a high graft failure rate 1-2 years following placement. These poor results, combined with increased association of these grafts with foreign body synovitis, tunnel osteolysis and premature osteoarthritis of the knee, led to a decrease in artificial ligament use nearly two decades ago [16]. In addition, Ventura et al. [25] demonstrated a high rate of degenerative osteoarthritis (OA) in all patients at a follow-up of 19 years following ACL reconstruction with polyethylene terephthalate artificial ligaments. The authors suggested that artificial ligaments likely contribute to the development of degenerative OA. Despite these discouraging results, artificial ligaments have recently regained renewed interest as a potential graft option for primary ACL reconstruction. Improved understanding in the design of artificial ligaments and good short-term results appear to be promising for new grafts, but we should be cautious not to be guided solely by this renewed enthusiasm. Therefore, only high-quality controlled studies with long-term follow-up should determine whether artificial ligaments have become an effective and safe graft option and, most importantly, do no harm to patients.

To ensure a high safety and efficiency level of new medical products, Malchau et al. [12] proposed a stepwise introduction of new medical devices to the orthopaedic market. Based on this concept, new medical products must pass stringent preclinical study evaluation on a small patient population, followed by three positive clinical investigations in a larger population. This concept allows close evaluation of long-term adverse reactions and potential complications that might occur. Many medical products, however, become available to the commercial market without ever being tested thoroughly and independently. All too often, the safety assessment(s) of these products by international health agencies is founded on the concept of "substantial equivalence". Substantial equivalence embodies the concept that if a new product or component is found to be substantially equivalent to an existing product or component, it can be treated in the same manner with respect to safety. These controversies over the definition and application of substantial equivalence are highlighted when one examines the harm that unfolded with artificial Metal-on-Metal (MM) hip implant [22]. The MM hip implant was released to orthopaedic markets worldwide without ever being clinically tested, since it was regarded to be substantially equivalent to existing artificial hip implant devices. In 2010, the MM hip implant was subsequently recalled from the market 5 years after its introduction, due to a failure rate of $12.5 \%$ [3, 23].

The revision rate of the MM hip implant increased to nearly $50 \%$ at 6 years after market introduction. Additionally, biological Adverse Local Tissue Reactions (ALTR) were found around MM implants such as pseudotumors, soft tissue distraction, as well as infiltration of lymphocytes and plasma cells. These side effects, sometimes referred to as adverse reaction to metal debris (ARMD), were thought to represent an immunological response to accelerated wear particles of the MM bearings $[11,20]$. Apart from these local side effects, several studies found 10- to 1,000-fold increases in cobalt ( $\mathrm{Co}$ ), chromium (Cr) and molybdenum (Mo) levels in the blood from patients with MM implants [26]. Whether these increased metal ion levels will ultimately result in harmful systematic effects remains unclear at this time. At the time of removal from the market, the MM hip implant was implanted in nearly 100,000 patients worldwide. While this is not the only example of a product receiving approval with negative outcomes reported later, it does serve as a frank reminder that new products, whether substantially equivalent or not, must be thoroughly evaluated both preclinically and clinically before use is allowed.

\section{Conflict of interest}

The medical device industry can work closely with physicians and surgeons in the product development, postdevelopment studies and clinical trials. These relationships should, however, be fully disclosed and regulated to ensure they are free of market-driven practices. Furthermore, research should be performed to the highest ethical standards and not be compromised by potential conflicts of interest. In Lancet, Lyman et al. [7] compared the differences between industry-sponsored and independently funded studies in 136 randomized trials investigating the treatment for multiple myeloma. This review found that industry-sponsored studies overall had a lower level of evidence compared to independently funded studies. Furthermore, industry-sponsored studies claimed innovative products were more beneficial than did independently funded studies. Additionally, the fact that a study sponsor can influence the reported findings or mask potentially harmful findings of negative side effects cannot be ignored. This was the case when genetically engineered bone morphogenetic protein- 2 product was introduced early in the 
year 2000 for bone grafting during anterior lumbar fusions. Between 2000 and 2009, 13 studies were conducted on this product, each of which reported positive results and no adverse effects associated with its use. During this time, bone morphogenetic protein-2 product has been implanted in over 500,000 patients. Nearly a decade after the first study was published, a group of independent researchers questioned the original results with comparably higher complication rates independently reported in the literature [5]. This group found the occurrence of adverse events to be 10-50 times higher than what was originally reported. Furthermore, the 13 studies that systematically failed to report on adverse events were found to have received industry funding on the order of \$12-\$16 million each [5]. An editorial written in an issue of the Spine Journal dedicated to this topic summarized: "It harms patients to have biased and corrupted research published ..." and "It harms patients to have unaccountable special interests permeate medical research" [4]. The actions of the researchers involved in these studies and the industries that were implicated highlight how financial interest in a multi-billion dollar product can potentially skew the values inherent to patient safety and above all underscores the need for independent research and funding in providing critical outcome data in relation to the so-called innovative products.

Evidence-based medicine and the influence of marketing

Evidence-based medicine (EBM), or the judicious use of the best available clinical evidence combined with the clinical expertise of the physician and the expectations and values of the patient [19], should be the fundamental principle for decision-making in the diagnosis and treatment of individual patients. The concept of evidence-based medicine, however, may itself be susceptible to bias, as all three pillars [(1) available evidence, (2) clinical expertise and (3) expectation and values of the patient] may be influenced by marketing campaigns by medical industry and non-scientific publications in the media. Particularly when the available evidence for a new "promising" product is low, the clinical expertise of the physician and the expectations of the patients may solely be founded on marketing and media reports. How new orthopaedic treatments can be popularized in public health is succinctly described by John A. Bergfeld with a so-called Orthopaedic Triad. According to this triad, a treatment may easily be popularized when the following three "factors" are combined: "a famous surgeon (1) who treats a famous athlete (2) with an untested treatment (3)" [9]. A recent example of a popularized treatment (modality) according to this triad is surrounding the use of the autologous orthobiologics in the field of orthopaedic surgery and sports medicine $[1,6]$.

Orthobiological treatment is the inclusion of biology and biochemistry in the development of skeletal and soft tissue healing. Popular orthobiological treatment modalities include platelet-rich plasma (PRP), autologous conditioned serum (ACS) and stem cells. Several of these modalities have been tagged as the "standard of care" and often assumed as a "fix" or "cure all" solution by both physicians and patients. Nevertheless, it should be expected that treatments of any kind undergo thorough investigations on a basic science and clinical level before widespread application in humans. In reality, these treatments are often implemented in daily practice based on the drive of popular culture and the positive mainstream media while there is insufficient scientific evidence to heretofore justify their clinical use as a standard of care.

PRP is produced from autologous whole blood using a standard, commercially available centrifugation system to yield a platelet concentrate which is elevated above baseline values. In the preparation process, the relative concentration of approximately 1,100 different growth factors is not controlled, and the ultimate composition of PRP remains unknown [14]. Lastly, it has been shown that not all growth factors are beneficial to all types of injures and/or effective at all time points [21]. Recently, Mazzocca et al. [14] showed that many variables, such as the system used for preparation, platelet activation, patient age and the time of day that the blood is drawn, affect the composition of PRP. Additionally, Foster et al. [8] evaluated the use of PRP and reported a lack of controlled clinical trials that provide a high level of evidence, since most of the studies were basic science studies and case reports reporting the use of PRP-related products without long-term follow-up. Overall, despite the lack of scientific evidence supporting the use of PRP, and the unpredictable and irreproducible studies supporting it, keen marketing campaigns and positive reports in the media have led widespread clinical use and a subsequent to a quickly increasing market value, which is estimated to be $\$ 126$ million by 2016 [13].

\section{Discussion}

Processes involved in the creation and testing of medical devices must be scrutinized to ensure safe and effective products are designed with the primary objective being increased benefit and no short- or long-term harm to patients. In addition, careful attention should be given as to how "innovation" itself is defined. In this regard, Prof. Ezekiel Emanuel of the University of Pennsylvania advocates, “... we need to stop glorifying every new technology 
as an innovation. "New" matters only when it's proved better than we had before..."

Without doubt, investigators should strive to report both positive and negative results in their research. In reality, researchers tend to be biased towards publishing positive results and neglecting to report negative results. Interestingly, an analysis by Dr. John P.A. Ioannidis of Stanford University underscored that while positive and negative trials are conducted in the same amount of time, it is indeed the negative trials that take an additional 2-4 years to be published. It is therefore not a question of whether these negative results are being reported, but rather an issue of the amount of barriers that exist to physicians and patients being informed of potential complications.

High-quality randomized controlled clinical trials are the "gold standard" by which innovative medical devices or interventions are evaluated for safety and clinical efficacy. The results of these trials must be evaluated and compared to the current standard of care without bias from marketing or industry. These trials must also be sufficiently powered to detect the effects of the intervention at the targeted tissue level and more importantly must include patient-relevant outcome measures to demonstrate that the new technology actually has a positive impact on the life of the patient. Perhaps, journals should refuse publication of case reports claiming positive results with novel treatments and require appropriately designed studies to investigate the benefits and harms of therapeutic interventions. Beyond this, even in high level of evidence publications, readers should critically appraise scientific publications in order to identify potential strengths, shortcomings and/or biases in study design, reporting of data and drawing conclusions. Again, it is equally, if not more, important to report negative results in these studies than it is to report positive results associated with innovative medical devices.

\section{References}

1. Alsousou J, Thompson M, Hulley P, Noble A, Willett K (2009) The biology of platelet-rich plasma and its application in trauma and orthopaedic surgery: a review of the literature. J Bone Jt Surg Br 91:987-996

2. Aronson JK, Ferner RE, Hughes DA (2012) Defining rewardable innovation in drug therapy. Nat Rev Drug Discov 11:253-254

3. Bernthal NM, Celestre PC, Stavrakis AI, Ludington JC, Oakes DA (2012) Disappointing short-term results with the DePuy ASR XL metal-on-metal total hip arthroplasty. J Arthroplasty 27: 539-544

4. Carragee EJ, Ghanayem AJ, Weiner BK, Rothman DJ, Bono CM (2011) A challenge to integrity in spine publications: years of living dangerously with the promotion of bone growth factors. Spine J 11:463-468

5. Carragee EJ, Hurwitz EL, Weiner BK (2011) A critical review of recombinant human bone morphogenetic protein-2 trials in spinal surgery: emerging safety concerns and lessons learned. Spine J 11:471-491

6. Dhillon RS, Schwarz EM, Maloney MD (2012) Platelet-rich plasma therapy-future or trend? Arthritis Res Ther 14:219

7. Djulbegovic B, Lacevic M, Cantor A, Fields KK, Bennett CL, Adams JR, Kuderer NM, Lyman GH (2000) The uncertainty principle and industry-sponsored research. Lancet 356:635-638

8. Foster TE, Puskas BL, Mandelbaum BR, Gerhardt MB, Rodeo SA (2009) Platelet-rich plasma: from basic science to clinical applications. Am J Sports Med 37:2259-2272

9. Kolata G (2011) As sports medicine surges, hope and hype outpace proven treatments. New York Times, New York

10. Legnani C, Ventura A, Terzaghi C, Borgo E, Albisetti W (2010) Anterior cruciate ligament reconstruction with synthetic grafts. A review of literature. Int Orthop 34:465-471

11. Mahendra G, Pandit H, Kliskey K, Murray D, Gill HS, Athanasou $\mathrm{N}$ (2009) Necrotic and inflammatory changes in metal-on-metal resurfacing hip arthroplasties. Acta Orthop 80:653-659

12. Malchau H, Bragdon CR, Muratoglu OK (2011) The stepwise introduction of innovation into orthopedic surgery: the next level of dilemmas. J Arthroplasty 26:825-831

13. Matava MJ (2012) Platelet-rich plasma: the next big thing? Commentary on an article by Ujash Sheth, BHSc, et al.: "Efficacy of autologous platelet-rich plasma use for orthopaedic indications: a meta-analysis". J Bone Jt Surg Am 94:e25

14. Mazzocca AD, McCarthy MB, Chowaniec DM, Cote MP, Romeo AA, Bradley JP, Arciero RA, Beitzel K (2012) Platelet-rich plasma differs according to preparation method and human variability. J Bone Jt Surg Am 94:308-316

15. Mulford JS, Chen D (2011) Anterior cruciate ligament reconstruction: a systematic review of polyethylene terephthalate grafts. ANZ J Surg 81:785-789

16. Olson EJ, Kang JD, Fu FH, Georgescu HI, Mason GC, Evans CH (1988) The biochemical and histological effects of artificial ligament wear particles: in vitro and in vivo studies. Am J Sports Med 16:558-570

17. Reitsma AM, Moreno JD (2002) Ethical regulations for innovative surgery: the last frontier? J Am Coll Surg 194:792-801

18. Reitsma AM, Moreno JD (2005) Ethics of innovative surgery: US surgeons' definitions, knowledge, and attitudes. J Am Coll Surg 200:103-110

19. Sackett DL, Rosenberg WM, Gray JA, Haynes RB, Richardson WS (1996) Evidence based medicine: what it is and what it isn't. BMJ 312:71-72

20. Schmalzried TP (2009) Metal-metal bearing surfaces in hip arthroplasty. Orthopedics 32. doi:10.3928/01477447-20090728-06

21. Shen W, Li Y, Zhu J, Schwendener R, Huard J (2008) Interaction between macrophages, TGF-beta1, and the COX-2 pathway during the inflammatory phase of skeletal muscle healing after injury. J Cell Physiol 214:405-412

22. Siebel T, Maubach S, Morlock MM (2006) Lessons learned from early clinical experience and results of 300 ASR hip resurfacing implantations. Proc Inst Mech Eng H 220:345-353

23. Smith AJ, Dieppe P, Vernon K, Porter M, Blom AW (2012) Failure rates of stemmed metal-on-metal hip replacements: analysis of data from the National Joint Registry of England and Wales. Lancet 379:1199-1204

24. Suter LG, Paltiel AD, Rome BN, Solomon DH, Golovaty I, Gerlovin H, Katz JN, Losina E (2011) Medical device innovation-is "better" good enough? N Engl J Med 365:1464-1466

25. Ventura A, Terzaghi C, Legnani C, Borgo E, Albisetti W (2010) Synthetic grafts for anterior cruciate ligament rupture: 19-year outcome study. Knee 17:108-113

26. von Schewelov T, Sanzen L (2010) Catastrophic failure due to aggressive metallosis 4 years after hip resurfacing in a woman in her forties-a case report. Acta Orthop 81:402-404 